\title{
IMPACT OF TRAINING ON HEMODYNAMIC PARAMETERS MEASURED DURING ANTI-G MANEUVERS
}

Lech KOPKA, Ewelina ZAWADZKA-BARTCZAK

Military Institute of Aviation Medicine, Department of Physiology, Warsaw, Poland

Source of support: Own sources

Author's address: L. Kopka, Military Institute of Aviation Medicine. Department of Physiology, Krasinskiego 54/56 Street, 01-755 Warsaw, Poland, e-mail: Ikopka@wiml.waw.pl, ezawadzka@wiml.waw.pl

Introduction: Physical training for pilots has been a focus of attention for a long period of time now. In this publication we assess the cardiovascular response to a series of anti-G straining maneuvers (AGSM) at baseline and after 2 months of muscle straining exercises and performing anti-G respiratory maneuvers (AGRM).

Methods: The study included 10 men - five F-16 pilots and five students from the Polish Air Force Academy in Dęblin. The following parameters were recorded: heart rate (HR), systolic blood pressure (SBP), diastolic (DBP) and mean (MAP), stroke volume (SV), cardiac output (CO), left ventricular work index (LVWI), total peripheral resistance (TPR), left ventricular ejection time (LVET), pre-ejection period (PEP).

Results: It was demonstrated that all parameters except for $\mathrm{CO}$ were significantly different after training.

Discussion: While performing AGSM, blood circulation becomes faster, which results in changes of the specific hemodynamic parameters. These parameters can provide information on the cardiovascular response to AGSM.

Conclusions: AGRM training in a"chair" position positively impacted response changes in hemodynamic parameters determining the $+\mathrm{Gz}$ acceleration tolerance. A non-invasive method of recording hemodynamic parameters using impedance may be useful for an assessment of the effectiveness of AGSM.

Keywords: + Gz acceleration, pilots, hemodynamics

Tables: 2 • Figures: 2 • References: 11 • Full-text PDF: http://www.pjamp.com • Copyright @ 2013 Polish Aviation Medicine Society, ul. Krasińskiego 54/56, 01-755 Warsaw, license WIML •Indexation: Index Copernicus, Polish Ministry of Science and Higher Education 


\section{INTRODUCTION}

Acceleration forces that act on a pilot in the $+G z$ axis (head to foot) cause displacement of soft tissues, organs (which are relatively mobile) and body fluids toward the lower part of the body. Blood redistribution and disturbed venous return to the right heart lead to hemodynamic disturbances [3]. They increase with the value of an acting G-force, decrease in the capacity of physiological compensatory mechanisms and reduction in the effectiveness of anti-G straining maneuvers (AGSM). Such maneuvers involve contracting skeletal and abdominal muscles with simultaneous attempted exhalation against partially (M-1 maneuver) or completely (L-1 maneuver) closed glottis. The stronger the contraction, the greater the pressure exerted on peripheral vessels. Pressure exerted on arteries impairs distribution of blood to the lower part of the body, promoting centralization of blond flow. Pressure on venous vessels facilitates venous return to the right heart. Increased mediastinal pressure due to forceful blocked exhalation is transferred to the heart and large vessels, increasing blood pressure and, in consequence, improving cerebral circulation [1,8,11]. Therefore, AGSM has a muscular and a respiratory component. Optimal performance of AGSM may improve the tolerance to the G-force exerted in the head to foot axis by +2 to $+4 \mathrm{~g}$ [6]. Prolonged forceful, blocked expiration may lead to dramatic decrease in venous return, blood pressure drop and loss of consciousness [9].

It has been shown that the muscular component is more useful for maintaining elevated mean blood pressure at the eye/brain level $[4,5]$. Significant improvement of $G$ tolerance through use of anti-G suits confirm the positive impact of indirect peripheral vessel compression as $+\mathrm{G} z$ acceleration increases.

Due to the above mentioned facts, great amount of attention is paid to pilot physical training. It is also worth noting that anti-G respiratory maneuvers are much more exhausting if performed while the $G$ acceleration is acting. Muscles participating in the inspiratory phase are then working against the G-force vector, which requires greater effort in stretching the chest.

Previous training of Air Force Academy cadets and pilots in proper performance of AGSM was based on visual assessment. Likewise, previously used method of measuring the skeletal muscle contraction strength (during dynamic pressure on the pedals of a measuring device) does not allow for assessing the influence of static muscle contraction on peripheral vascular resistance and blood flow. Only the results of expensive tests performed in a centrifuge would give some insight into individual effects achieved through such training. Also, it seems obvious that even technically well done AGSM may be performed better after proper training.

In another publication [5] we demonstrated positive influence of static training of lower body muscles (it involves maintaining a "chair" position) with simultaneous AGRM exercises on the $+\mathrm{Gz}$ tolerance. The impact of such training on the changes in hemodynamic parameters that occur while performing a series of AGSMs under stationary conditions has not been studied. It may be expected that improved $+G z$ tolerance observed after training was a result of positive hemodynamic changes. The aim of this work was the following: evaluation of changes in hemodynamic parameters during AGSM series performance under stationary conditions before and after training.

\section{METHODS}

The group consisted of ten men aged 20-39 years (mean 28.1 years), 170-188 cm tall (mean $174.8 \mathrm{~cm}$ ), weighing $62.5-92 \mathrm{~kg}$ (mean $75.8 \mathrm{~kg}$ ), including 5 experienced F-16 pilots and 5 secondyear cadets from the Polish Air Force Academy in Dęblin.

None of the subjects smoked, there was no history of syncope or other important morbidities. No one abused alcohol or other drugs, nobody took any medication. No abnormalities in echoand electrocardiographic examination, complete blood count or electrolyte levels were noted in our subjects. Results of spirometry, chest x-ray and other examinations routinely performed for periodic medical check-ups were unremarkable.

Subjects performed the anti-G respiratory maneuvers in a "chair" position, which resembled a situation of sitting on a chair without a seat (with their back resting against the wall), as presented in Figure 1.

Training was conducted according to the following scheme: a series of 10 AGRMs with 15-second breaks between each series performed up to the point of leg muscle fatigue precluding further exercise, once or twice a day for a period of 2 months.

During that time subjects also performed other physical exercise (fitness, running, walking, swimming, cycling, etc.) as before.

Hemodynamic measurements were performed in the morning, in the same room, under identical 


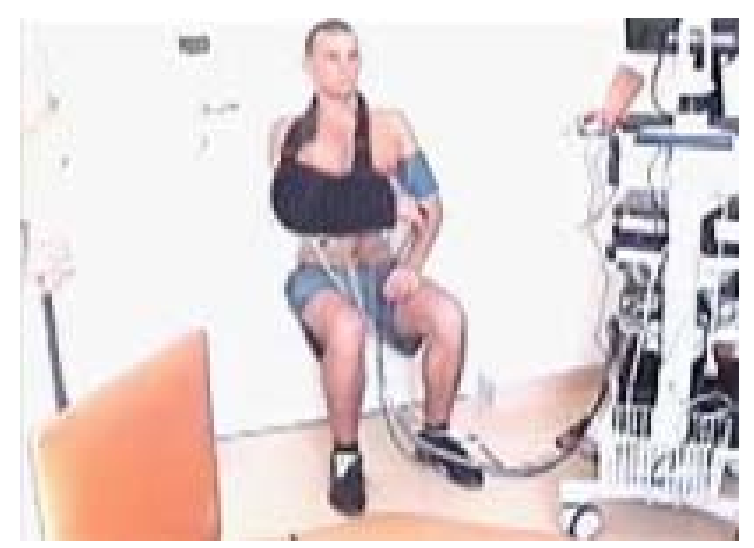

Fig. 1. Body position during anti-G respiratory maneuvers is called a "chair" position.

conditions with regard to level of noise, moisture and surrounding temperature (air-conditioning). All subjects reported for examinations well rested, after a good night sleep, did not take any stimulants or pharmacological agents before the test.

Assessment of circulatory system involved the following recorded parameters:
1. Heart rate (HR).

2. Blood pressure: systolic (sBP), diastolic (dBP), mean (MAP).

3. Stroke volume (SV).

4. Cardiac output (CO).

5. Left Ventricular Work Index (LVWI).

6. Total peripheral resistance (TPR).

7. Left Ventricular Ejection Time ( LVET).

8. Pre-Ejection Period (PEP).

Beat-to-beat recording of hemodynamic was performed using a TASK FORCE MONITOR device by CNS system.

Mean values of hemodynamic parameters registered during three series of anti-G maneuvers lasting 15 seconds before and after training were compared. A subject performed AGSM during each one of these series. Breaks between the series lasted 15 seconds and were meant for rest (like the breaks between subsequent ROR program intervals in a centrifuge).

AGSM series is defined as separate AGSMs performed immediately one after another. After

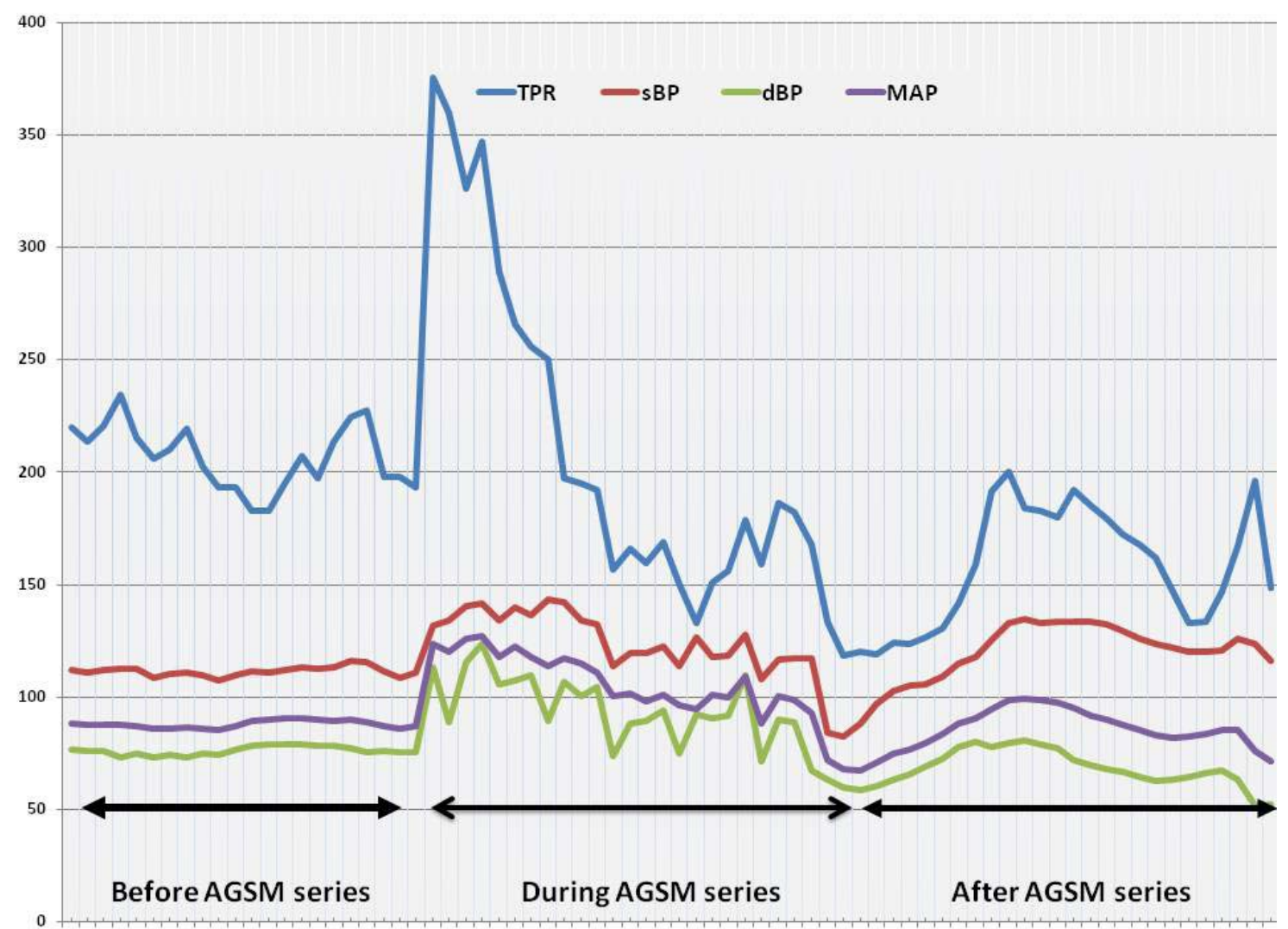

Fig. 2a. Graphic representation of changes in: total peripheral resistance (TPR), systolic blood pressure (sBP), diastolic blood pressure (dBP) and mean arterial pressure (MAP). 
a quick expiration, the subject performs a rapid (lasting about 1s) deep breath followed by forced, blocked expiration lasting about 3s. etc.

Above tests were performed on a chair positioned identically to a chair in F-16 and the subjects assumed the same position as during centrifuge tests. Their feet rested on rudder pedals. Left forearm was placed on an armrest positioned at a heart level (requirement of measurement method). Right forearm rested on the second armrest in such a way so that a sensor placed on subject's fingers (which took BP measurements using pletysmography) was at a heart level.

Statistical significance was assessed using a two-way student's t-test for dependent samples with $\mathrm{p}<0.05$.

\section{RESULTS}

An example of changes in hemodynamic parameters recorded during AGSM series (beat-tobeat recordings) in one of the subjects is displayed as graphs in Figure 2a and $2 \mathrm{~b}$.

One should note that during a 15-second break before subsequent AGSM series, the recorded hemodynamic parameters did not return to initial values. Therefore, every successive series was commenced in a hemodynamic situation that differed from the baseline.

Comparison of mean values of hemodynamic parameters recorded in a study group during 3 successive AGSM series before and after training is presented in Table 1.

Mean cumulative values of hemodynamic parameters acquired during all 3 AGSM series before and after training are presented in Table 2.

It was demonstrated that all assessed parameters except for CO significantly changed after training in a "chair" position.

\section{DISCUSSION}

Advances in medical technology made it possible to perform non-invasive, simultaneous recording of hemodynamic parameters and autonomic nervous system important for monitoring of patient condition and progress of treatment. In this work we recorded hemodynamic parameters (using impedance) during a series of AGSM maneuvers (thus, under dynamic conditions). The study

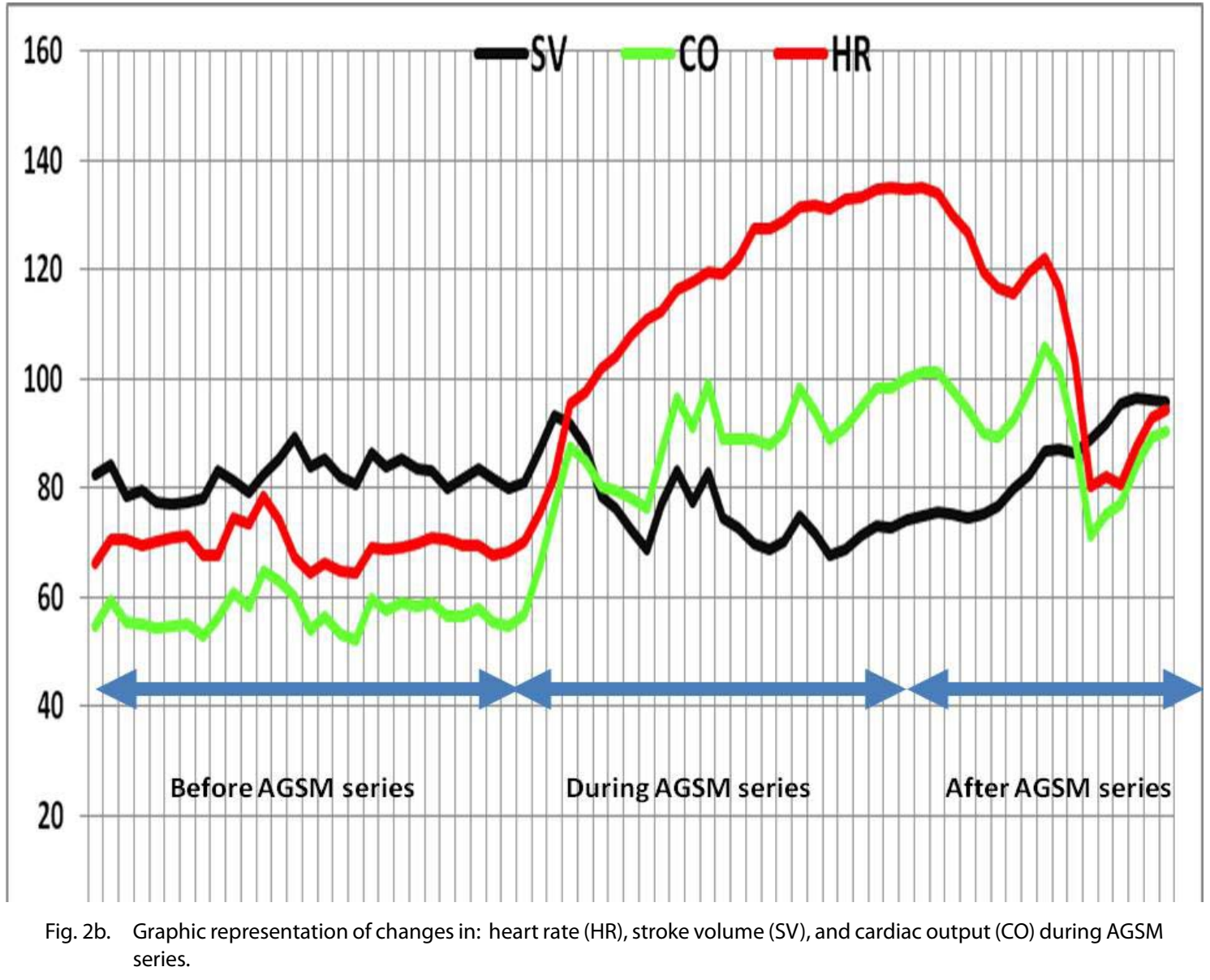


L. Kopka and al. - Impact of training...

Tab. 1. Mean values of hemodynamic parameters recorded during successive AGSM series before and after training.

\begin{tabular}{|c|c|c|c|c|c|c|c|c|c|c|}
\hline \multicolumn{2}{|c|}{ AGSM } & \multicolumn{3}{|c|}{ Series 1} & \multicolumn{3}{|c|}{ Series 2} & \multicolumn{3}{|c|}{ Series 3} \\
\hline & & Mean & SD & $\mathbf{P}$ & Mean & SD & $\mathbf{P}$ & Mean & SD & $\mathbf{P}$ \\
\hline \multirow{2}{*}{ HR (bpm) } & BT & 107.2 & 18 & \multirow{2}{*}{$0.001 *$} & 106.7 & 14.86 & \multirow{2}{*}{$0.000^{*}$} & 102.7 & 16.91 & \multirow{2}{*}{$0.000^{*}$} \\
\hline & AT & 114.4 & 23.67 & & 118.5 & 23.49 & & 119 & 24.31 & \\
\hline \multirow{2}{*}{ sBP $(\mathrm{mmH})$} & BT & 127 & 20.89 & \multirow{2}{*}{$0.000^{*}$} & 139.5 & 20.03 & \multirow{2}{*}{$0.000^{*}$} & 150.5 & 24.29 & \multirow{2}{*}{0.16} \\
\hline & AT & 145.1 & 20.23 & & 152.1 & 18.78 & & 153.2 & 14.91 & \\
\hline \multirow{2}{*}{$\mathrm{dBP}(\mathrm{mmHg})$} & BT & 93.9 & 20.08 & \multirow{2}{*}{$0.000^{*}$} & 96.6 & 22.2 & \multirow{2}{*}{$0.000^{*}$} & 105.2 & 22.23 & \multirow{2}{*}{$0.002^{*}$} \\
\hline & AT & 107.7 & 20.03 & & 109.9 & 19.42 & & 111.1 & 17.17 & \\
\hline \multirow{2}{*}{ MAP (mmHg) } & BT & 107.5 & 20 & \multirow{2}{*}{$0.000^{*}$} & 114.8 & 20.46 & \multirow{2}{*}{$0.000^{*}$} & 123.6 & 22.15 & \multirow{2}{*}{0.077} \\
\hline & AT & 122.5 & 18.33 & & 125.8 & 16.79 & & 126.7 & 13.64 & \\
\hline \multirow{2}{*}{ SV (ml) } & BT & 86.8 & 17.58 & \multirow{2}{*}{$0.000^{*}$} & 99.8 & 15.7 & \multirow{2}{*}{$0.000^{*}$} & 98.5 & 15.69 & \multirow{2}{*}{$0.000^{*}$} \\
\hline & AT & 78.5 & 15.34 & & 89.2 & 20.12 & & 87.3 & 16.84 & \\
\hline \multirow{2}{*}{$\mathrm{CO}(\mathrm{I} / \mathrm{min})$} & BT & 9.1 & 1.72 & \multirow{2}{*}{0.092} & 10.5 & 1.56 & \multirow{2}{*}{0.107} & 10 & 1.73 & \multirow{2}{*}{0.369} \\
\hline & AT & 8.8 & 1.81 & & 10.2 & 1.68 & & 10.1 & 1.69 & \\
\hline \multirow{2}{*}{$\begin{array}{l}\text { TPR } \\
\text { (dyn *s/cm5) }\end{array}$} & BT & 954.6 & 289.89 & \multirow{2}{*}{$0.000^{*}$} & 872.5 & 237.98 & \multirow{2}{*}{$0.000^{*}$} & 993.5 & 250.18 & \multirow{2}{*}{0.646} \\
\hline & AT & 1130.9 & 292.49 & & 982.4 & 221.61 & & 1003.7 & 197.67 & \\
\hline \multirow{2}{*}{$\begin{array}{l}\text { LVWI } \\
\text { (kg m/m2) }\end{array}$} & BT & 6.6 & 1.25 & & 7.5 & 1.42 & & 7.6 & 1.38 & \\
\hline & AT & 7.0 & 1.51 & & 7.9 & 1.37 & & 8.1 & 1.28 & 0.002 \\
\hline IMCT /ms & BT & 249.2 & 30.48 & 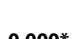 & 241.4 & 25.74 & Sarar & 246.3 & 30.37 & nemex \\
\hline LVET(III) & AT & 230.6 & 37.82 & 0.000 & 221.6 & 34.52 & 0.000 & 223.3 & 36.87 & 0.000 \\
\hline ו & BT & 101.9 & 16.62 & (م) & 96.8 & 15.13 & مas & 99.4 & 14.92 & \\
\hline PET (III) & AT & 108.2 & 15.36 & 0.000 & 100.5 & 14.77 & 0.013 & 100.3 & 16.79 & 0.334 \\
\hline
\end{tabular}

BT - before training

AT - after training

Tab. 2. Mean cumulative values of hemodynamic parameters acquired during three AGSM series before and after training.

\begin{tabular}{|c|c|c|c|c|}
\hline & AGSM & Mean & SD & $\mathbf{P}$ \\
\hline \multirow{2}{*}{ HR (bpm) } & BT & 105.53 & 2.47 & \multirow{2}{*}{0.000} \\
\hline & AT & 117.30 & 2.52 & \\
\hline \multirow{2}{*}{ sBP (mmHg) } & BT & 139.00 & 11.76 & \multirow{2}{*}{0.000} \\
\hline & AT & 150.13 & 4.39 & \\
\hline \multirow{2}{*}{ dBP $(\mathbf{m m H g})$} & BT & 98.57 & 5.90 & \multirow{2}{*}{0.000} \\
\hline & AT & 109.57 & 1.72 & \\
\hline \multirow{2}{*}{ MAP (mmHg) } & BT & 115.30 & 8.06 & \multirow{2}{*}{0.000} \\
\hline & AT & 125.00 & 2.21 & \\
\hline \multirow{2}{*}{ SV (ml) } & BT & 95.03 & 7.16 & \multirow{2}{*}{0.000} \\
\hline & AT & 85.00 & 5.71 & \\
\hline \multirow{2}{*}{$\mathrm{CO}(\mathrm{I} / \mathrm{min})$} & BT & 9.87 & 0.71 & \multirow{2}{*}{0.2} \\
\hline & AT & 9.70 & 0.78 & \\
\hline \multirow{2}{*}{ TPR (dyn * s/cm5) } & BT & 940.20 & 61.77 & \multirow{2}{*}{0.000} \\
\hline & AT & 1039.00 & 80.30 & \\
\hline \multirow{2}{*}{ LVWI (kg-m/m2) } & BT & 7.23 & 0.55 & \multirow{2}{*}{0.000} \\
\hline & AT & 7.67 & 0.59 & \\
\hline \multirow{2}{*}{ LVET (ms) } & BT & 245.63 & 3.94 & \multirow{2}{*}{0.000} \\
\hline & AT & 225.17 & 4.78 & \\
\hline \multirow{2}{*}{ PEP (ms) } & BT & 99.37 & 2.55 & \multirow{2}{*}{0.000} \\
\hline & AT & 103.00 & 4.50 & \\
\hline
\end{tabular}


group consisted of Polish Air Force Academy cadets and pilots, the great majority of who greatly benefitted from a 2-month AGRM training in a "chair" position with regard to $+\mathrm{Gz}$ tolerance improvement [5]. Selected hemodynamic parameters recorded during 3 AGSM series were analyzed before and after training.

It should be emphasized that values of recorded hemodynamic parameters physiologically undergo constant, slight changes which occur even during rest. During a series of performed AGSMs blood circulation becomes more dynamic. When considering theoretically the hemodynamic effect of each successive AGSM during the entire series one should refer to a hemodynamic state produced as a consequence of the previous AGSM, which is practically impossible as the end of one AGSM almost immediately becomes the beginning of another one. Thus, each subsequent AGSM begins in a hemodynamic state that significantly differs from the initial one. Even during 15-second brakes between three successive AGSM series recorded values of hemodynamic parameters did not return to baseline. For the above reasons we used mean values of hemodynamic parameters recorded in a given period of time when discussing the results of our work.

Theoretically, cumulative response of baroreceptors from the entire cardiovascular system is additionally influenced by unequal volumes of air in the lungs during successive AGSMs [7]. It has an impact on mediastinal pressure and therefore, amount of venous return, particularly during AGSMs performed in series. It is also worth noting that during non-invasive assessment of cardiovascular system under static conditions dynamic nature of some hemodynamic parameters (e.g. vessel diameter, density, viscosity, blood velocity and resistance) is neglected for the matter of simplicity and their number is reduced to optimal minimum. Thus, only average values of certain parameters are in fact recorded at a given time.

Physiological adaptation of the cardiovascular system to static physical effort mainly involved changes in the following parameters: HR, MAP, TPR and LVWI [1]. The most important parameter responsible for $+\mathrm{Gz}$ acceleration tolerance is MAP. This pressure value at a level of the eye determines G-tolerance of the body. Physiologically, mean blood pressure depends on TPR and CO [10]. It was shown in the present work that mean $\mathrm{SBP}, \mathrm{dBP}$ and MAP values recorded after three AGSM series were significantly higher after training. AGRM training in a "chair" position positively impacted the strength of skeletal and respiratory muscle contraction to such extent to allow for generating higher blood pressures during a series of anti-G maneuvers.

Total vascular resistance largely depends on the activity of sympathetic vascular fibers and local factors released by endothelium (causing vessel constriction or dilatation) [11]. Increased sympathetic activity in control stress tests performed after training is evidenced by, i.e. significant increase in mean HR and TPR values. Sympathetic activation also increases the strength of cardiac contraction and therefore, the force that the heart muscle had to counteract in order to eject the stroke volume to peripheral vessels had to be higher. After the training, the subjects were capable of greater and more effective muscular effort during performance of AGSM series. Therefore, mean LVWI value increased significantly during control stress tests.

Stroke volume is the difference between enddiastolic and end-systolic volumes. It is determined by, among other things, cardiac contractility, preload and afterload - relationship between contractility and preload is directly proportional and inversely proportional to afterload [11]. However, changes of mean SV values before and after training should not be analyzed separately from heart rate, which greatly influences this parameter and depends on many internal and external factors. Similar objection may apply to interpretation of a parameter, which is very important from the viewpoint of G-tolerance - systemic vascular resistance, which is known as afterload. Parameters such as, i.e. MAP, central venous pressure (if unknown, appropriate software uses a default value) and cardiac output (product of SV and HR) are used to calculate this value. Since SV is influenced by the above parameters, their changes automatically determine $\mathrm{CO}$ value, which did not change statistically after the training.

With proper cardiac function PEP (time from beginning of left ventricular depolarization to opening of the aortic valve) becomes prolonged due to decrease in venous return and, in consequence, end-diastolic volume of the left ventricle and its distension. More effective execution of AGRMs is probably responsible for prolonged mean values of this parameter after training. Forceful blocked expiration causes significant increase in intrathoracic pressure and thereby impedes venous return. Thus, the more effectively maneuver is performed, the greater the impediment.

It is known that LVET (time from aortic valve opening to its closing) shortens with decrease in venous return in the course of SV reduction. This relationship is in agreement with acquired results. After training, during the AGSM series mean SV val- 
L. Kopka and al. - Impact of training...

ues decreased significantly and LVET became shortened, while the value of PEP significantly increased.

It is worth noting that the range of hemodynamic changes occurring during AGSM series after training varied from an individual to individual in our study group, which might ensue from unequal physical fitness and not identical AGSM L-1 techniques.

Increase in the mean values of: TPR, LVWI, PEP, $H R, s B P, d B P$ and MAP observed in follow-up tests may be explained by the conducted training. Mean values of hemodynamic parameters achieved during AGSM series in a group of men, in whom training improved $+\mathrm{Gz}$ tolerance confirmed our theoretical expectations, which makes them more reliable.

Hemodynamic parameters that may have a beneficial influence on blood redistribution during AGSMs performed during high values of $+G z$ accelerations changed for the better. It was therefore demonstrated that determination of these parameters during dynamic tests using presented method might be useful in aviation medicine.

\section{CONCLUSIONS}

1. AGRM training in a "chair" position positively impacted response changes in hemodynamic parameters determining the $+G z$ acceleration tolerance.

2. A non-invasive method of recording hemodynamic parameters using impedance may be useful for an assessment of the effectiveness of AGSM.

\section{AUTHORS' DECLARATION:}

Study Design: Lech Kopka, Ewelina Zawadzka-Bartczak; Data Collection: Lech Kopka, Ewelina Zawadzka-Bartczak; Statistical Analysis: Lech Kopka, Ewelina Zawadzka-Bartczak; Manuscript Preparation: Lech Kopka, Ewelina Zawadzka-Bartczak; Funds Collection: Lech Kopka, Ewelina ZawadzkaBartczak. The Authors declare that there is no conflict of interest.

\section{REFERENCES}

1. Burton, R.R. (1986) A conceptual model for predicting pilot group G tolerance for tactical fighter aircraft. Aviat Space Environ Med. 57 (8). 733-744.

2. Green, N.D.C. (2006) Protection of long duration acceleration. In Rainford, D.J. \& Gradwell, D.P. (eds.) Ernsting's Aviation Medicine. London: Hodder Arnold.

3. Howard, P. (1997) Gravity and the circulation. Proc Royal Soc. London B. 199 (1137). 485-91.

4. Kobayashi, A., Kikukawa, A., Onozawa, A. (2002) Effect of muscle tensing on cerebral oxygen status during sustained high +Gz. Aviat Space Environ Med. 73 (6). 597-600.

5. Kopka, L., Zawadzka-Bartczak, E., Kopka, M. (2012) Influence of anti-G respiratory maneuver training in "chair" position on +Gz tolerance. Polski Przegląd Medycyny i Psychologii Lotniczej. 18 (3). 49-61.

6. Mac Dougall, J.D., Mc Kelvie, M.D., Moroz, D.E., Moroz, J.S., Buick, F. (1993) The effects of variations in the anti-G straining maneuver on blood pressure at $+G z$ acceleration. Aviat Space Environ Med. 64 (2). 126-131.

7. Mateika, J.H., DeMeersman, R.E., Kim, J. (2002) Effects of lung volume and chemoreceptor activity on blood pressure and R-R interval during the Valsalva maneuver. Clin Auton Res. 12 (1). 24-34.

8. Scott, J.M., Esch, B.T., Goodman, L.S., Bredin, S.S., Haykowsky, M.J., Warburton, D.E., (2007) Cardiovascular consequences of high-performance aircraft maneuvers: implications for effective countermeasures and laboratory-based simulations. Appl Physiol Nutr Metab. 32 (2). 332-9.

9. Shubrooks, S.J. Jr., Leverett, S.D. Jr (1973) Effect of the Valsalva maneuver on tolerance to +Gz acceleration. J Appl Physiol. 34 (4). 460-466. 
Original Article

10. Traczyk, W. \& Trzebski, A. (2001) (eds.) Fizjologia człowieka z elementami fizjologii stosowanej i klinicznej. Warszawa: PZWL.

11. Wood, E.H. \& Hallenbeck, G.A. (1946) Voluntary (self-protective) maneuvers which can be used to increase man's tolerance to positive acceleration. Fed Proc. 5 (1 Pt 2). 115.

Cite this article as: Kopka L, Zawadzka-Bartczak E: Impact of training on hemodynamic parameters measured during anti-G maneuvers. Pol J Aviat Med Psychol, 2013; 19(1): 13-20. 